\title{
ARTICLE Honokiol inhibits breast cancer cell metastasis by blocking EMT through modulation of Snail/Slug protein translation
}

\author{
Wen-die Wang ${ }^{1}$, Yue Shang ${ }^{1}, \mathrm{Yi} \mathrm{Li}^{1}$ and Shu-zhen Chen ${ }^{1}$
}

\begin{abstract}
Honokiol (HNK), an active compound isolated from traditional Chinese medicine Magnolia officinalis, has shown potent anticancer activities. In the present study, we investigated the effects of HNK on breast cancer metastasis in vitro and in vivo, as well as the underlying molecular mechanisms. We showed that HNK $(10-70 \mu \mathrm{mol} / \mathrm{L})$ dose-dependently inhibited the viability of human mammary epithelial tumor cell lines MCF7, MDA-MB-231, and mouse mammary tumor cell line 4T1. In the transwell and scratch migration assays, $\operatorname{HNK}(10,20,30 \mu \mathrm{mol} / \mathrm{L})$ dose-dependently suppressed the invasion and migration of the breast cancer cells. We demonstrated that HNK $(10-50 \mu \mathrm{mol} / \mathrm{L})$ dose-dependently upregulated the epithelial marker E-cadherin and downregulated the mesenchymal markers such as Snail, Slug, and vimentin at the protein level in breast cancer cells. Using a puromycin incorporation assay, we showed that HNK decreased the Snail translation efficiency in the breast cancer cells. In a mouse model of tumor metastasis, administration of HNK (50 mg/kg every day, intraperitoneal (i.p.), 6 times per week for 30 days) significantly decreased the number of metastatic 4T1 cell-derived nodules and ameliorated the histological alterations in the lungs. In addition, HNKtreated mice showed decreased Snail expression and increased E-cadherin expression in metastatic nodules. In conclusion, HNK inhibits EMT in the breast cancer cells by downregulating Snail and Slug protein expression at the mRNA translation level. HNK has potential as an integrative medicine for combating breast cancer by targeting EMT.
\end{abstract}

Keywords: honokiol; breast cancer cell; epithelial-mesenchymal transition (EMT); Snail; Slug

Acta Pharmacologica Sinica (2019) 40:1219-1227; https://doi.org/10.1038/s41401-019-0240-x

\section{INTRODUCTION}

Breast cancer is the most common malignant disease and second leading cause of cancer-related death among females worldwide, and more than $90 \%$ of deaths are attributed to invasive breast cancer [1, 2]. Although increased breast screening, newer aggressive therapeutics, and reduced use of hormone replacement therapy have decreased the death rate, metastatic breast cancer remains a major challenge $[1,2]$.

Epithelial-mesenchymal transition (EMT) is involved in cancer invasion and metastasis by producing cells with a more motile and invasive phenotype [3-5]; this transition features the loss of Ecadherin and acquisition of $\mathrm{N}$-cadherin and vimentin [6]. EMT is orchestrated by a set of transcription factors including Snail, Slug, Zinc Finger E-Box Binding Homeobox 1 (ZEB1), Zinc Finger E-Box Binding Homeobox 2 (ZEB2), and Twist, which repress epithelial markers and induce mesenchymal markers. The expression of these key transcription factors is tightly regulated at the levels of transcription, translation and protein stability [6]. Thus, key factors controlling EMT have been identified as potential targets to prevent and treat metastatic cancer [7-9].

Honokiol (HNK) is a bioactive component isolated from the Chinese traditional herb Magnolia officinalis, which has been demonstrated to be a potent anticancer drug with activity in inducing apoptosis and inhibiting the growth of various cancer cells [10-12]. Recent studies have shown that HNK exhibits potent anticancer activities, including inhibition of cancer cell invasion
[13-15]. Avtanski et al. reported that HNK effectively inhibits EMT in breast cancer cells, thereby establishing HNK as a promising active compound against breast cancer $[14,16]$. Nevertheless, the effect of HNK in breast cancer remains elusive.

In this study, we examined the effects of HNK on breast cancer cell metastasis and EMT through in vitro and in vivo experiments. In addition, the underlying mechanism for EMT's inhibition by HNK was also evaluated.

\section{MATERIALS AND METHODS}

Antibodies and reagents

Rabbit anti-Snail, -Slug, -E-cadherin, -vimentin, -phospho-elF2a, and -elF2a monoclonal antibodies were obtained from Cell Signaling Technology (Danvers, MA, USA). The primary anti-Snail antibody used for immunohistochemistry was produced by Abcam (Cambridge, UK). The mouse antipuromycin monoclonal antibody was obtained from Merck Millipore (Darmstadt, Germany). Mouse monoclonal antibodies against $\beta$-actin were obtained from ZSGB-BIO (Beijing, China). HNK was purchased from Shanghai Ziyi-reagent Company (Shanghai, China), dissolved in dimethyl sulfoxide (DMSO) as a $100 \mathrm{mmol} / \mathrm{L}$ stock solution, and stored at $-20^{\circ} \mathrm{C}$. All stock solutions were diluted with RPMI-1640 or with other medium to obtain the indicated final concentration before the experiments were performed.

\footnotetext{
${ }^{1}$ Institute of Medicinal Biotechnology, Chinese Academy of Medical Sciences \& Peking Union Medical College, Beijing 100050, China
}

Correspondence: Shu-zhen Chen (bjcsz@imb.pumc.edu.cn)

Received: 4 January 2019 Accepted: 25 April 2019

Published online: 24 June 2019 
Cell culture

The human mammary epithelial tumor cell lines MCF7 and MDAMB-231 and mouse mammary tumor cell line 4T1 were obtained from American Type Culture Collection (ATCC, Manassas, VA, USA). The non-tumorigenic mammary epithelial MCF10A cells were obtained from the National Infrastructure of Cell Line Resource (Beijing, China) and were cultured in DMEM/F12 (1:1) medium supplemented with $5 \%$ horse serum (FBS, Gibco, Grand Island, NY, USA), $10 \mu \mathrm{g} / \mathrm{mL}$ of insulin, $0.5 \mu \mathrm{g} / \mathrm{mL}$ of hydrocortisone, $20 \mathrm{ng} / \mathrm{mL}$ of EGF, and $100 \mathrm{ng} / \mathrm{mL}$ of cholera toxin. The MCF7 and MDA-MB231 cell lines were maintained in Dulbecco's modified Eagle's medium (DMEM, HyClone, Logan, UT, USA), and 4T1 cells were maintained in RPMI-1640 medium (HyClone); both types of media were supplemented with $10 \%$ fetal bovine serum (FBS, Gibco, Grand Island, NY, USA) and the cells were cultured in a $37^{\circ} \mathrm{C}$ incubator with $5 \% \mathrm{CO}_{2}$.

Cell survival analysis

Cell viability was evaluated using the 3-(4,5-dimethylthiazol-2-yl) -2,5-diphenyl tetrazolium bromide (MTT) assay (Solarbio, Beijing, China). Cells were seeded in a 96-well plate at a density of $5 \times 10^{3}$ cells/well and were treated with the indicated concentrations of HNK. After $48 \mathrm{~h}$ of incubation, the cells were treated with MTT solution $(10 \mu \mathrm{L}, 5 \mathrm{mg} / \mathrm{mL})$ for $4 \mathrm{~h}$. Subsequently, the supernatant was discarded. The formazan crystals were solubilized with $150 \mu \mathrm{L}$ of DMSO. The absorbance was examined at $570 \mathrm{~nm}$ using a microplate reader (Thermo Fisher Scientific Inc., Waltham, MA, USA), and cell viability was determined by comparing the absorbance of treated cells with that of the control cells. All $\mathrm{MTT}$ experiments were performed in triplicate and were repeated for at least 3 times.

\section{Scratch migration assay}

Cells were plated into six-well plates for migration assays and were allowed to grow overnight to confluence. A sterile pipette tip was used to create a scratch in the cell layer, and then the wounded cell monolayer was washed 3 times with PBS to remove floating cells and debris. Medium containing 3\% FBS and/or HNK was added to the wells, and the plates were maintained at $37^{\circ} \mathrm{C}$. The identical location in each plate was photographed at 0 and $24 \mathrm{~h}$. All experiments were repeated 3 times under each condition.

In vitro migration assay

The in vitro cell migration capabilities were determined using the Transwell assay as described previously [17]. Briefly, $5 \times 10^{4}$ cells were suspended in $100 \mu \mathrm{L}$ of serum-free medium and were placed into the upper compartment of chambers containing polycarbonate filters (Transwell; $8 \mu \mathrm{m}$ pore size; Corning Incorporated, Corning, NY, USA). The lower compartment was filled with $600 \mu \mathrm{L}$ of complete medium supplemented with $10 \%$ FBS in the absence or presence of HNK. After $24 \mathrm{~h}$, the inserts were fixed in methanol and stained with crystal violet. The non-migrating cells on the upper surface of the filter were carefully removed with a cotton swab. The migrated cells on the lower surface of the filter were photographed using an inverted microscope (Leica Microsystems, Wetzlar, Germany). Finally, the membrane was solubilized into $33 \%$ acetic acid, and the absorbance at $570 \mathrm{~nm}$ was measured with a microplate reader. The experiments were conducted in triplicate.

\section{Western blotting analysis}

Immunoblotting was performed as described previously [18]. Equal amounts of protein lysate were separated by SDS-PAGE and were electrophoretically transferred to polyvinylidene fluoride (PVDF) membranes. After blocking, the membranes were incubated with primary antibodies at $4{ }^{\circ} \mathrm{C}$ overnight, followed by incubation with the secondary antibody for $1 \mathrm{~h}$ at room temperature. Finally, the Western blot signals were detected using an ECL detection system (ProteinSimple, CA, USA).

Immunoprecipitation and puromycin incorporation assay To measure the intensity of Snail translation, $1 \mu \mathrm{mol} / \mathrm{L}$ of puromycin (Solarbio, Beijing, China, diluted in PBS) was added in the culture medium and the cells were incubated for $1 \mathrm{~h}$ at $37^{\circ} \mathrm{C}$ and $5 \% \mathrm{CO}_{2}$. The cells were then harvested and immunoprecipitated with anti-Snail antibody. IP was performed according to standard protocol. Briefly, after cold PBS washing, the MCF7 cells $(3 \times 10 \mathrm{~cm}$ plate dishes) were lysed with IP lysis buffer containing protease inhibitors for $30 \mathrm{~min}$ on ice. Next, lysed samples were centrifuged at $14000 \times$ for $20 \mathrm{~min}$ at $4{ }^{\circ} \mathrm{C}$. After discarding the precipitate, the supernatants were incubated with normal lgG or anti-Snail antibody and protein $A / G$ magnetic beads (Thermo Fisher Scientific, Massachusetts, USA) overnight at $4{ }^{\circ} \mathrm{C}$. The precipitated complexes were washed with lysis buffer 5 times. The proteins were eluted with SDS sample buffer and then boiled for $10 \mathrm{~min}$ and analyzed by SDS-PAGE. Puromycin antibodies were used for Western blot analysis.

RNA extraction and quantitative RT-PCR

Total RNA was extracted using the Total RNA Purification Kit according to the manufacturer's instructions (Shanghai Feijie, Shanghai, China). RNA quality was assessed using the NanoDrop Lite UV-Vis spectrophotometer (Kaiao, Beijing, China). cDNA was synthesized using ReverTra Ace qPCR RT Master Mix (Toyobo Co., Ltd., Osaka, Japan). The mRNA levels of Snail, Slug, and glyceraldehyde 3-phosphate dehydrogenase (GAPDH) were measured via quantitative RT-PCR using SYBR Premix ExTaq (Takara, Tokyo, Japan) and the ABI 7500HT Fast Real-time PCR system (Applied Biosystems, Foster City, CA, USA). The following primers were used: human Snail 5'-CCAGACCCACTCAGATGTCAA-3' (forward) and 5'-GGACTCTTGGTGCTTGTGGA-3' (reverse); mouse Snail 5'-GTCTGCACGACCTGTGGAAA-3' (forward) and 5'-G GTCAGCAAAAGCACGGTTG-3' (reverse); human and mouse Slug 5'-GCTTCAAGGACACATTAGAACTCA-3' (forward) and 5'-CATTC TGGAGAAGGTTTTGGAGC-3' (reverse); human GAPDH 5'-ACAACT TTGGTATCGTGGAAGG-3' (forward) and 5'-GCCATCACGCCACAGT TTC-3' (reverse); and mouse GAPDH 5'-AGGTCGGTGTGAACGG ATTTG- $3^{\prime}$ (forward) and $5^{\prime}$-TGTAGACCATGTAGTTGAGGTCA-3' (reverse). GAPDH was used for normalization. The data were analyzed using the $\Delta \Delta \mathrm{Ct}$ method, and each assay was performed in duplicate.

\section{In vivo lung metastasis model}

Female BALB/c nude mice (6-8 weeks old) were purchased from SPF Biotechnology (Beijing, China) and were maintained under specific pathogen-free conditions. All animal experiments were conducted in accordance with the institutional guidelines, which are in compliance with national and international laws and policies. 4T1 cells were injected into the mice (six per group) via the tail vein at a concentration of $1 \times 10^{6}$ cells $/ 0.1 \mathrm{~mL}$ PBS per mouse. After 1 week, the mice were treated with i.p. injections of Intralipid or $50 \mathrm{mg} / \mathrm{kg}$ every day of HNK in $20 \%$ Intralipid (Sigma-Aldrich, St. Louis, MO, USA) six times per week. Thirty days later, the mice were sacrificed, and the lungs were removed and fixed in $10 \%$ formalin. The lung metastatic nodules were examined macroscopically and subjected to hematoxylin and eosin (H\&E) staining and immunohistochemical analysis. For immunohistochemistry, paraffin-embedded sections were deparaffinized in xylene and rehydrated in graded alcohol. The slides were boiled in $10 \mathrm{mmol} / \mathrm{L}$ sodium citrate buffer, $\mathrm{pH}$ 6.0, for antigen retrieval. Staining was performed using the Enhanced Polymer Detection Kit (ZSGB-BIO, Beijing, China) according to the manufacturer's protocol. DAB was used as a peroxidase substrate. 
Statistical analysis

All the experiments were repeated independently at least three times. The quantitative data are presented as the means $\pm S D$. Two-tailed Student's $t$-test was used to evaluate the differences between the groups. $P<0.05$ indicates statistical significance.

\section{RESULTS}

HNK inhibits breast cancer cell viability

To evaluate the effect of HNK on cancer cell survival, MDA-MB-231, MCF7, and $4 \mathrm{~T} 1$ breast cancer cell lines and non-tumorigenic MCF10A cells were exposed to various concentrations of HNK $(0-70 \mu \mathrm{mol} / \mathrm{L})$ for $48 \mathrm{~h}$. An MTT assay was used to examine the cell viability. The results showed that HNK reduced cell proliferation in a concentration-dependent manner except in MCF10A cells (Fig. 1b), as previously reported [19]. To specifically examine whether HNK inhibits EMT and the metastatic properties of breast cancer cells, 30 or $40 \mu \mathrm{mol} / \mathrm{L}$ of $\mathrm{HNK}$ was chosen for further experiments.

HNK suppresses cell migration and invasion

The migration of breast cancer cells in response to HNK was measured using Transwell migration and scratch migration assays. Compared with MDA-MB-231 and 4T1 cells, MCF7 cells showed low metastatic behavior (Fig. 2a). When MDA-MB-231 and 4T1 cells with high metastatic potential were treated with HNK for $24 \mathrm{~h}$, their migratory activity was markedly decreased, occurring through the movement of cells into the scarred region, compared with the control group (Fig. 2b, c). The same results were observed in the Transwell migration assay (Fig. 2d, e), consistent with the results of previous studies $[14,20]$. Taken together, these data
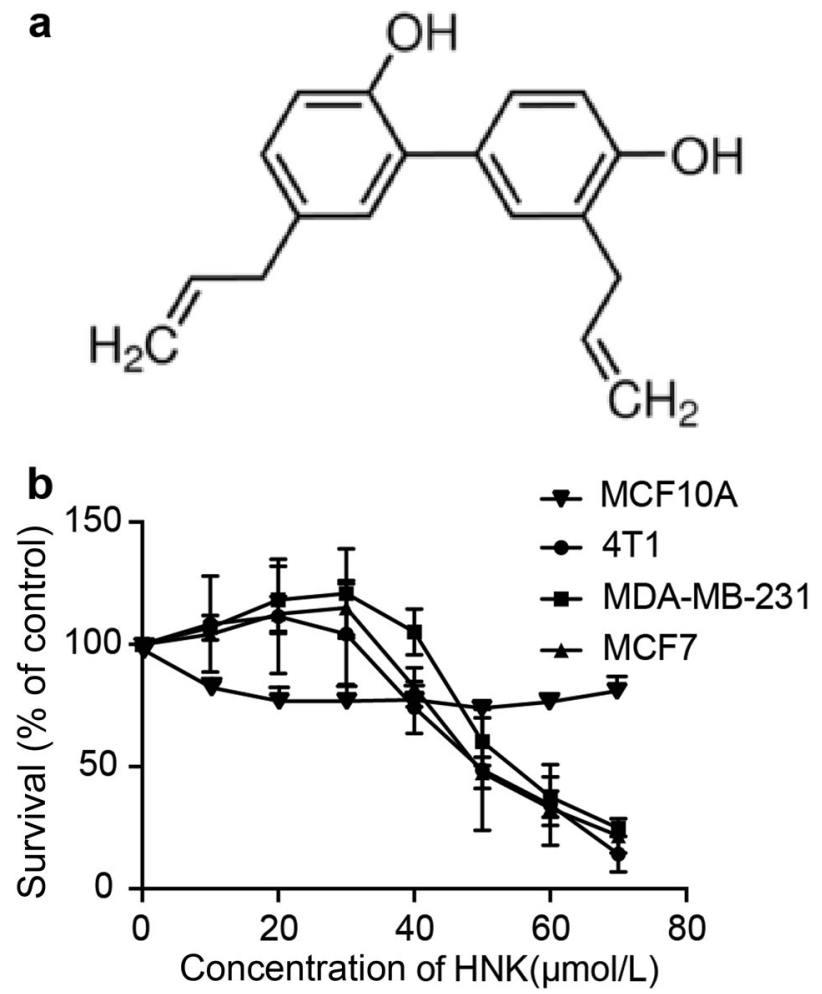

Fig. 1 HNK reduces breast cancer cell viability. a Chemical structure of HNK. b MCF10A, 4T1, MDA-MB-231, and MCF7 cells were treated with various concentrations of HNK for $48 \mathrm{~h}$. Cell viability was investigated by the MTT assay. The data are shown as the means \pm SD. $n=3$ strongly demonstrated that HNK treatment resulted in effective inhibition of migration in breast cancer cells.

HNK affects the expression levels of EMT-associated transcription factors Snail/Slug in breast cancer cells

EMT is involved in the invasion and metastasis of various cancer cells. To better understand whether HNK is involved in this process, epithelial and mesenchymal markers [21-23] were assessed by Western blot analysis. As shown in Fig. 3a, HNK markedly downregulated endogenous Snail, Slug, and vimentin expression and upregulated $\mathrm{E}$-cadherin expression at the protein level in MDA-MB-231, MCF7, and 4T1 cells (Fig. 3a). As master EMT inducers, Snail and Slug dictate the induction of EMT by targeting E-cadherin and vimentin [24-26]. Furthermore, when cells were treated for different times, the western blotting results showed that HNK decreased Snail and Slug expression levels from $12 \mathrm{~h}$ to $24 \mathrm{~h}$ (Fig. $3 \mathrm{~b}$ ). The results suggested that HNK can reverse EMT via the downregulation of Snail and Slug in breast cancer cell lines.

HNK inhibits the in vivo metastasis of breast cancer cells To further validate our in vitro findings, an in vivo study was conducted in a BALB/c nude mouse 4T1 metastasis model. Although $4 \mathrm{~T} 1$ cells metastasize to various primary organs, including the lung, liver, bone, and brain, we focused on lung metastases in this study. We collected lung tissues from tumorbearing mice for histological analysis, 30 days after the injection of $4 \mathrm{~T} 1$ cells. The size and number of nodules in the lungs were visualized and measured. Treatment with $50 \mathrm{mg} / \mathrm{kg}$ of HNK clearly reduced the number and size of metastatic nodules in the lungs, but did not significantly affect the body weight compared with vehicle treatment (Fig. 4a-c). As shown in Fig. 4d, histological alterations in the lungs of mice in the HNK treatment group were ameliorated. In addition, immunohistochemical assays were performed to examine the Snail and E-cadherin levels. The Snail expression level was decreased and the Ecadherin expression level was increased in the HNK-treated group compared with that in the control group (Fig. 4e), consistent with the in vitro analysis.

HNK has no downregulated effect on Snail/Slug mRNA expression or protein stability in breast cancer cells

The inhibition of in vivo metastasis by HNK prompted us to elucidate the mechanisms underlying the antimetastasis effect of HNK. Because Snail and Slug protein levels were decreased in HNK-treated cells, reverse transcription-quantitative PCR (RT-qPCR) analysis was applied to analyze the mRNA expression levels of Snail and Slug in MDA-MB-231, MCF7, and 4T1 cells. However, the mRNA expression of Snail and Slug was transiently upregulated in MCF7 and MDA-MB-231 cells after exposure to HNK. In addition, no significant difference in the Snail mRNA levels in 4T1 cells was observed after exposure to HNK (Fig. 5a). In addition, we observed extremely low Slug mRNA levels in $4 \mathrm{~T} 1$ cells, consistent with the immunoblotting results, and explained the instability of the results of Slug in 4T1 cells (Fig. 5a, e). We then ascertained the effect of HNK on Snail and Slug protein stability in the presence of cycloheximide (CHX), which inhibits translation (Fig. 5c-e). Densitometric quantification of immunoblots showed that Snail and Slug protein levels were decreased rapidly after CHX treatment, but HNK did not change the half-life of Snail or Slug protein in cells.

HNK mediates elF2a phosphorylation and translation inhibition in breast cancer cells

Having obtained unexpected results regarding Snail/Slug mRNA and protein levels in HNK-treated cell lines, we further investigated the mechanisms by which HNK decreases Snail/Slug protein levels and assessed whether HNK generally affects the translation 


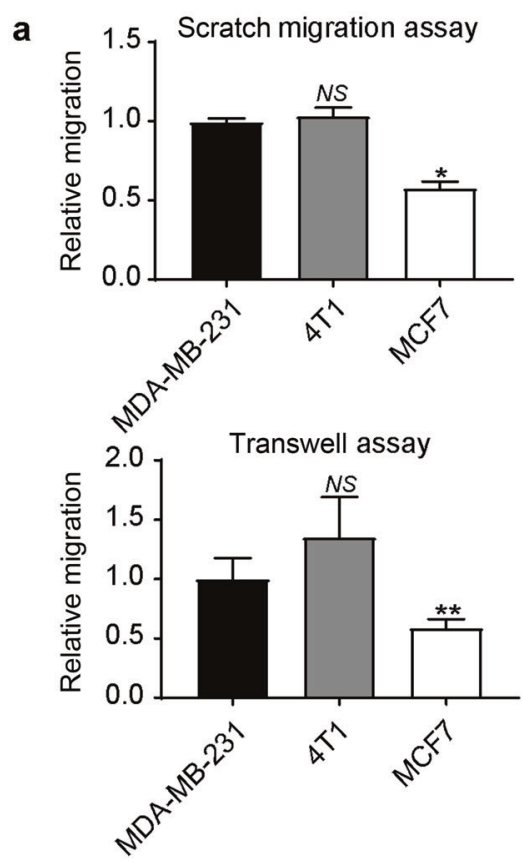

b

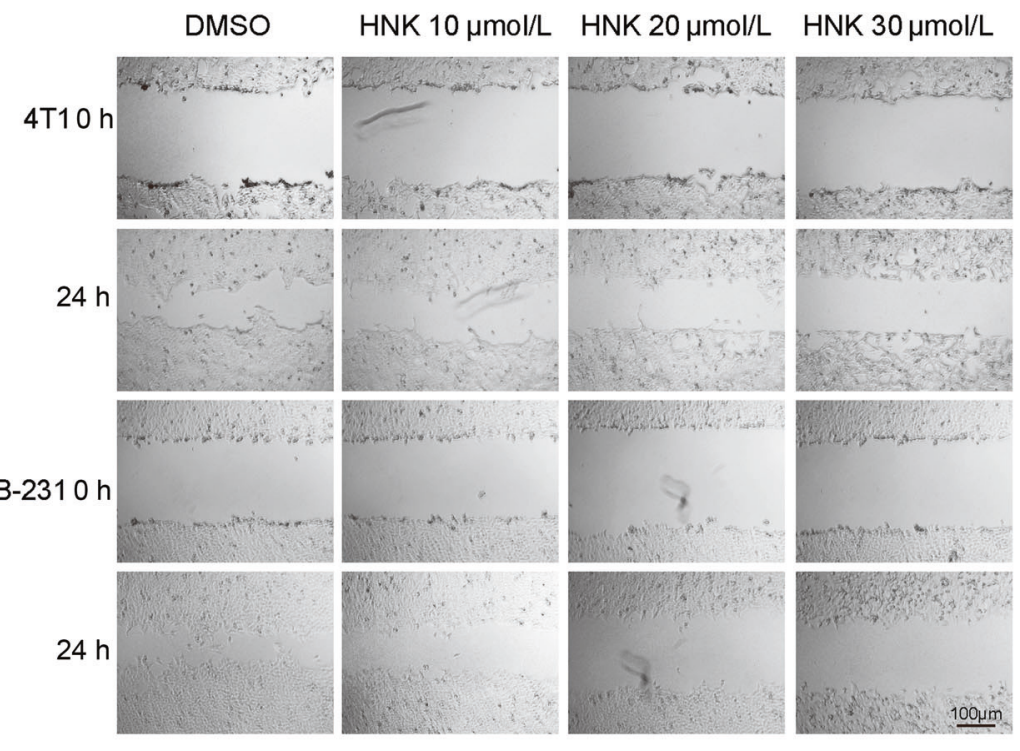

d
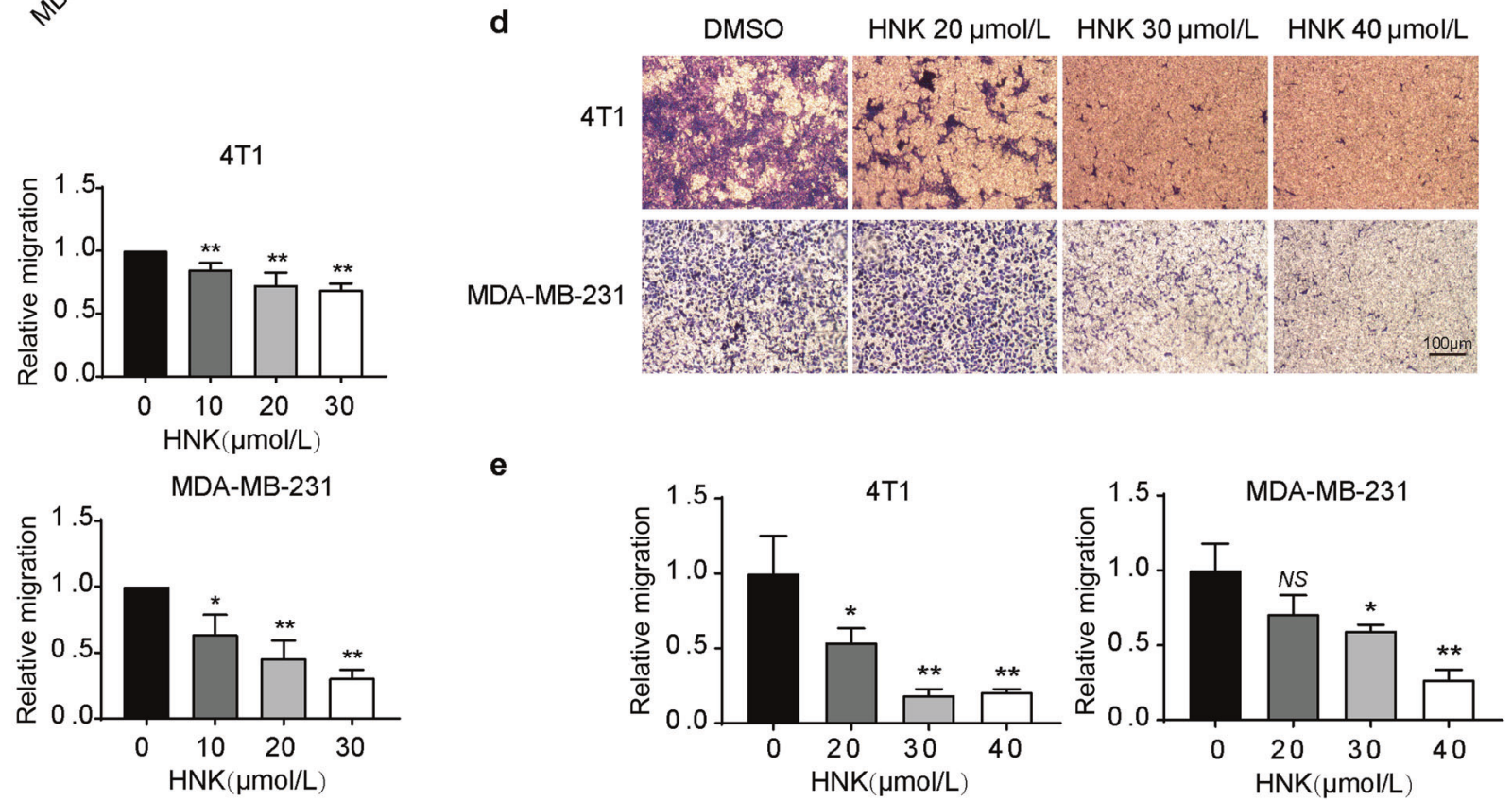

Fig. 2 HNK suppresses the invasion and migration of breast cancer cells. a Quantitative measurement of the Transwell and scratch migration assays of MDA-MB-231, 4T1, and MCF7 cells. b, c 4T1 and MDA-MB-231 cells were treated with HNK (0-30 $\mu \mathrm{mol} / \mathrm{L})$ and were subjected to a scratch migration assay $(40 \times)$. d, e Transwell assays of $4 \mathrm{~T} 1$ and MDA-MB-231 cells cultured with HNK $(0-40 \mu \mathrm{mol} / \mathrm{L})$ as indicated for $24 \mathrm{~h}$. Representative images are shown and the results were obtained with a microplate reader $(40 \times)$. The data are shown as the means \pm SD. $n=3$. NS, No significance, ${ }^{*} P<0.05,{ }^{*} P<0.01$ compared with the indicated group

of Snail and Slug mRNA. We first established a time course of 0,1 , $2,4,6$, and $8 \mathrm{~h}$ of HNK treatment in MCF7 cells. Snail protein levels started to decrease at $2 \mathrm{~h}$ and further decreased at 4 and $6 \mathrm{~h}$ after HNK treatment. The phosphorylation of eukaryotic initiation factor $2 \mathrm{~A}$ (elF2a) started to increase at $1 \mathrm{~h}$ and continued to increase from 1 to $8 \mathrm{~h}$ after HNK treatment. Similar results were observed in MDA-MB-231 and 4T1 cells (Fig. 6a). Based on these results, we chose the 8-h timepoint for HNK treatment in further studies. Indeed, increased elF2a phosphorylation revealed that HNK might reduce protein translation. Next, the global translation rate of MCF7 cells was measured by puromycin incorporation, which was marginally decreased in HNK-treated MCF7 cells compared with control cells (Fig. 6b). Furthermore, Snail was immunoprecipitated from cell extracts and detected by Western blotting using the antipuromycin antibody. As shown in Fig. 6b, puromycin labeling was dramatically decreased after HNK treatment. The decreased protein translation suggested that HNK might inhibit Snail mRNA translation. These observations supported our hypothesis that HNK-mediated downregulation of Snail and Slug proteins occurred at the transcriptional level.

\section{DISCUSSION}

The antitumor activity of HNK has been reported in various preclinical models and it has shown to target multiple facets of signal transduction [27, 28]. HNK can attenuate Ras-mediated 

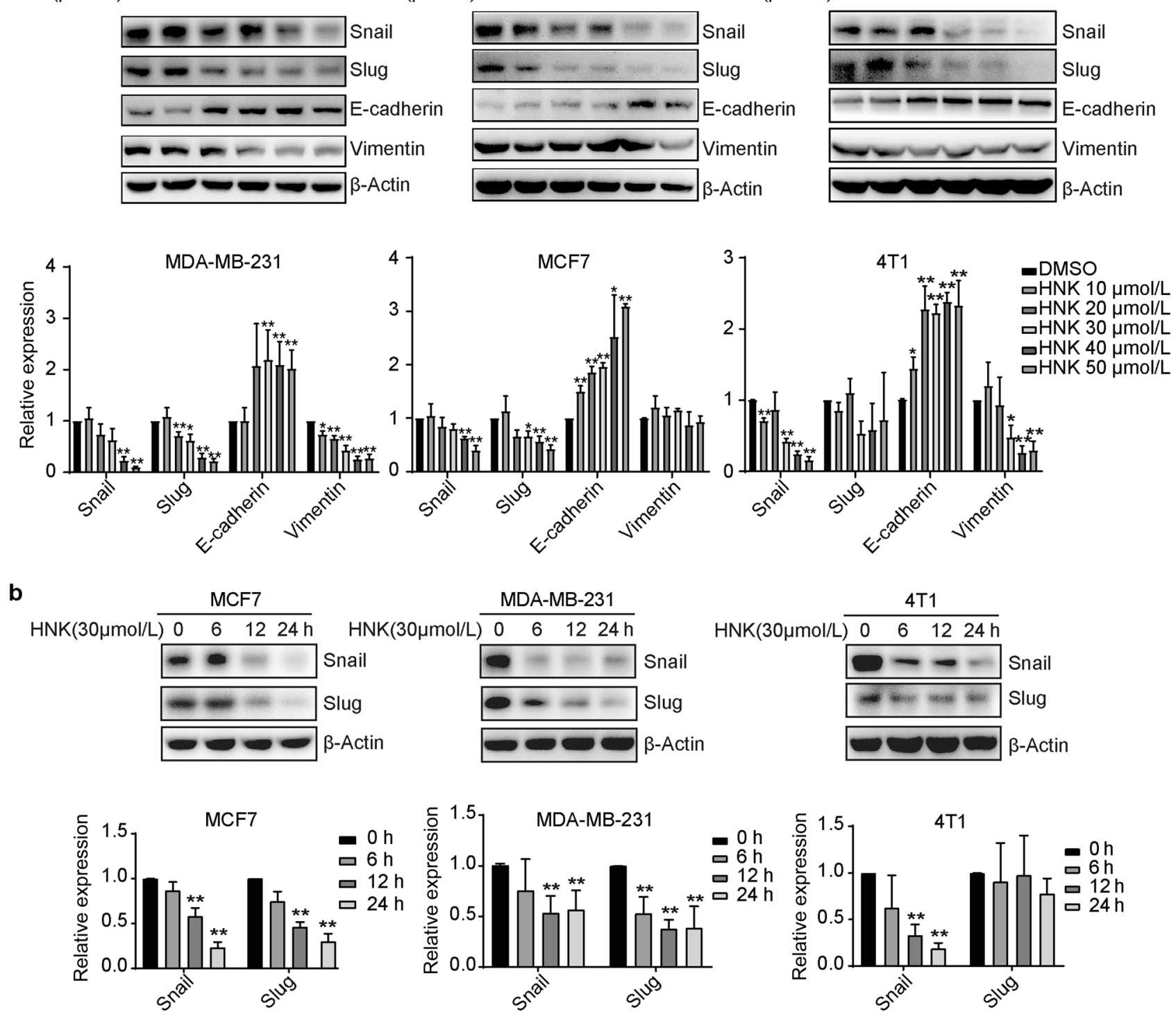

Fig. 3 HNK inhibits Snail and Slug expression in breast cancer cells. a The cells were treated with the indicated concentrations of HNK for $24 \mathrm{~h}$ and cell lysates were analyzed by Western blotting using the indicated antibodies. $\beta$-Actin was used as a loading control. The band densities of proteins were quantified by ImageJ software. b 4T1, MDA-MB-231, and MCF7 cells were treated with HNK for different periods of time (0, 6 , 12 , or $24 \mathrm{~h}$ ) and were analyzed by Western blotting. The band densities of proteins were calculated using ImageJ software. ${ }^{*} P<0.05,{ }^{* *} P<0.01$ compared with the control group

oncogenic pathways [29], eliminate stem-like cells, induce apoptosis, and suppress Wnt/ $\beta$-catenin signaling in oral cancer cells [30]. Recently, researchers have reported that HNK-induced ER stress and calpain-II activation are involved in the regulation of cancer cell migration and invasion [15]. However, the mechanisms by which HNK inhibits invasion and metastasis have not been fully clarified. In the present study, we investigated the role of HNK in breast cancer migration and invasion in vivo and in vitro and explored the underlying molecular mechanisms. We found that HNK effectively inhibited the invasion of breast cancer cells in vitro; this result was corroborated by its effect in the in vivo analyses of breast cancer cell lung metastasis. These findings were consistent with previous reports that HNK inhibits the invasion and migration of breast cancer cells $[16,20]$. As a major process underlying tumor metastasis, EMT is characterized by the loss of cell-cell adhesion and increased cell motility and invasion. We observed that HNK inhibited the motility of breast cancer cells. Consistently, HNK upregulated the epithelial marker E-cadherin and downregulated mesenchymal markers such as Snail, Slug, and vimentin in breast cancer cells.

Snail and Slug are members of the Snail superfamily of zincfinger transcription factors. Notably, Snail and Slug are the main effectors of many EMT inducers and repressors because of their crucial roles in the induction of EMT [31]. Snail and Slug were found to induce EMT progression by downregulating E-cadherin expression and enhancing vimentin expression [26, 32, 33]. Hence, we focused on the regulation of Snail and Slug protein expression and found that HNK treatment decreased the expression of these proteins in a dose- and time-dependent manner. Yao et al. suggested that the suppression of Snail and Slug in HNK-treated 


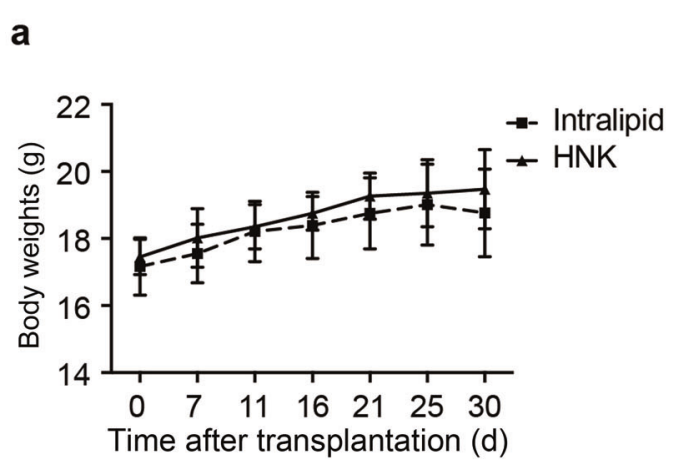

d
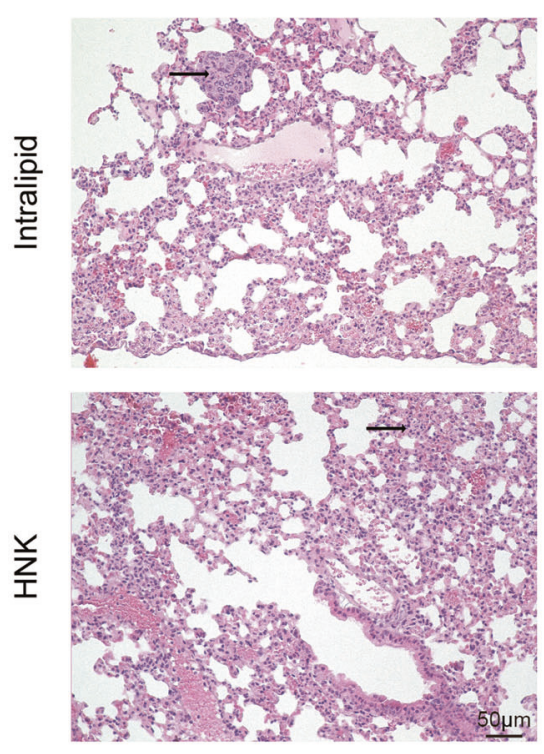

e

\section{b}
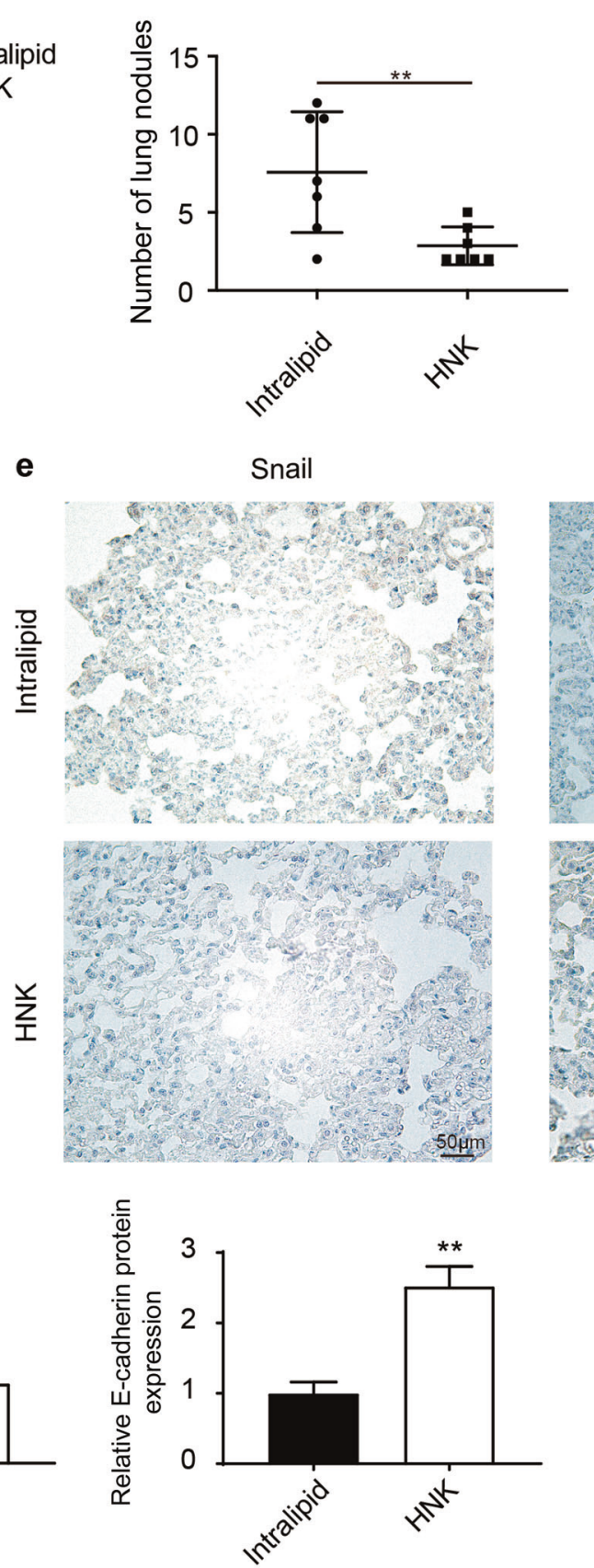

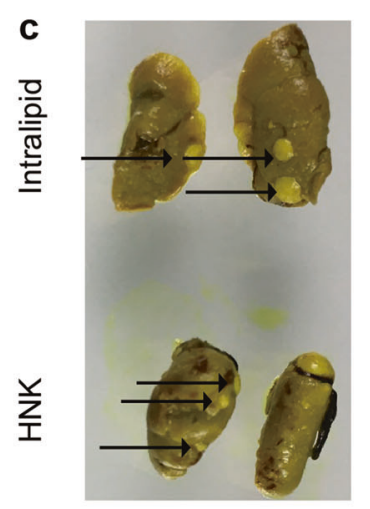

E-cadherin
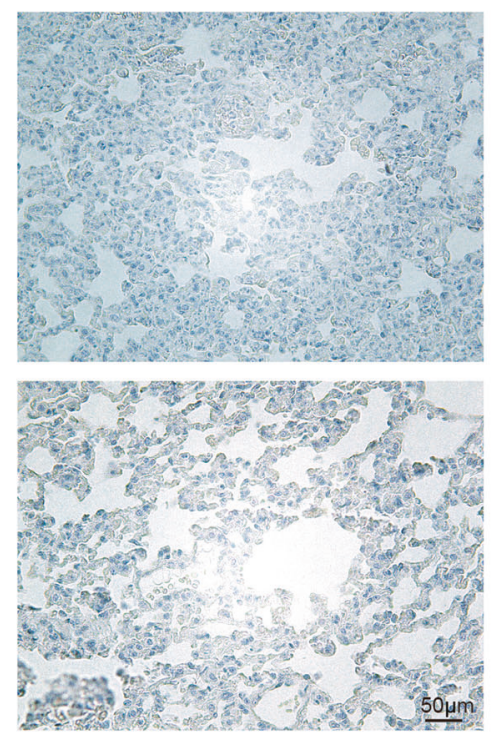

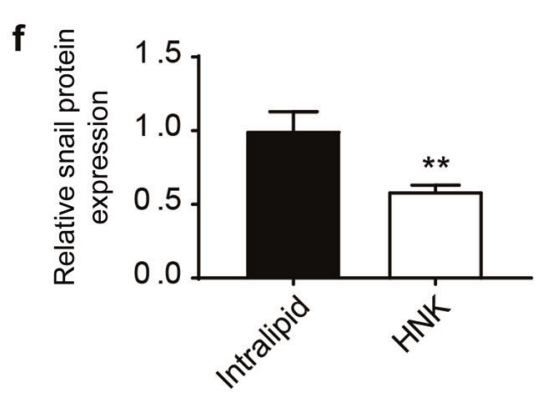

Fig. 4 HNK inhibits lung metastasis of breast cancer cells in nude mice. $\mathbf{a}$, $\mathbf{b}$ Body weight and number of lung metastasis nodules in $4 \mathrm{~T} 1$ tumor-bearing mice administered Intralipid (control) or HNK, $n=6 .{ }^{* *} P<0.01$. c Representative photos of lung metastasis are shown for each group. The arrows indicate the metastasis foci. $\mathbf{d}$ Metastatic lung nodules were confirmed by hematoxylin $\&$ eosin staining $(200 \times)$. The arrows indicate metastasis foci. e, $\mathbf{f}$ Immunohistochemical assays showed Snail and E-cadherin expression levels in the control and HNK-treated groups $(400 \times)$. The bar diagram shows the relative quantification evaluated by densitometry. ${ }^{* *} P<0.01$ compared with the control group

side population (SP) cells might occur via the $\mathrm{Wnt} / \beta$-catenin signaling pathway [30]. In addition, a study on gastric tumors demonstrated that the downregulation of Snail by HNK may occur through the regulatory effect of Tpl2 [34]. However, the precise mechanism by which HNK downregulates Snail and Slug in breast cancer cells remains to be determined.

Snail and Slug expression is regulated at different levels, including the transcriptional, translational, and posttranslational levels. Many signaling molecules, including FGF, Wnt, and TGF, regulate Snail at the transcriptional level [35-37]. Chronic hypoxia specifically upregulates Slug mRNA and protein expression in prostate cancer cells [38]. In addition, ATM was shown to regulate Snail stabilization by the phosphorylation of Serine 100, thus promoting tumor invasion and metastasis. However, baicalein can induce Snail and Slug ubiquitination by stimulating the interaction between GSK3 $\beta$ and Snail or Slug [39]. LKB1 is a potential suppressor of prostate cancer cell metastasis that promotes Snail protein degradation by enhancing the interaction between the E3 

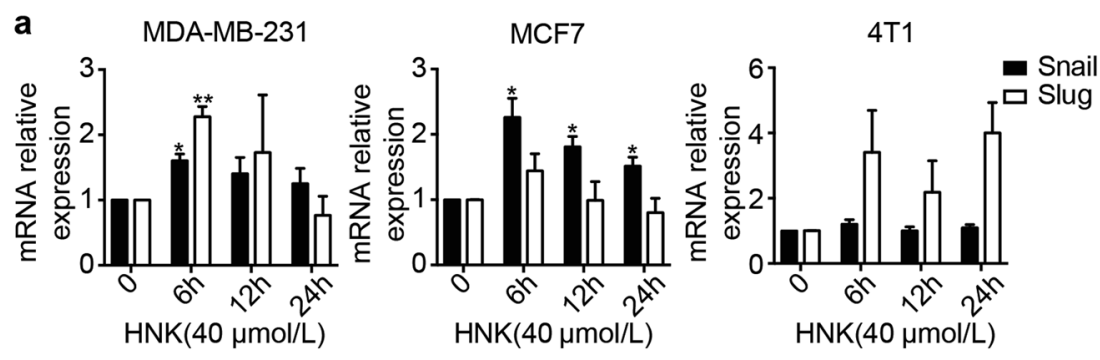

b

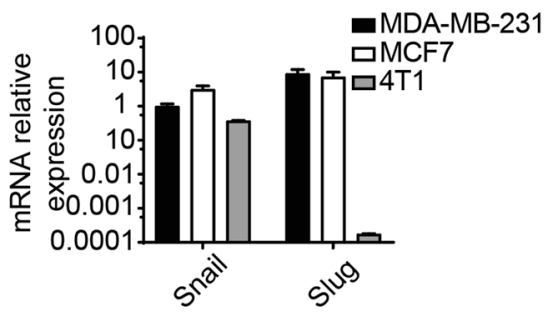

C

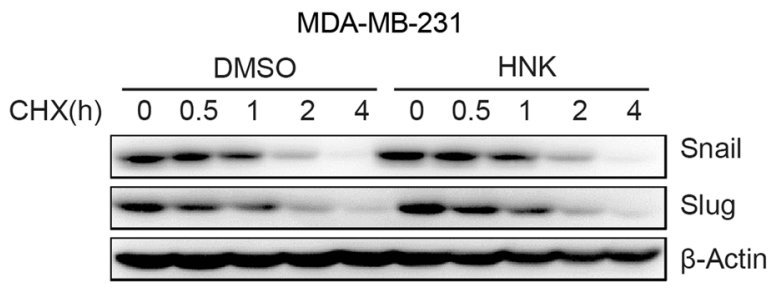

d

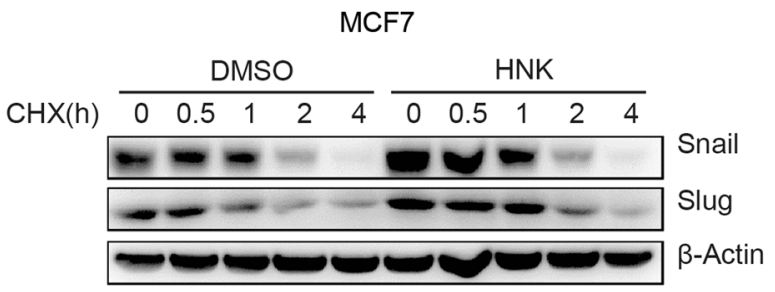

e

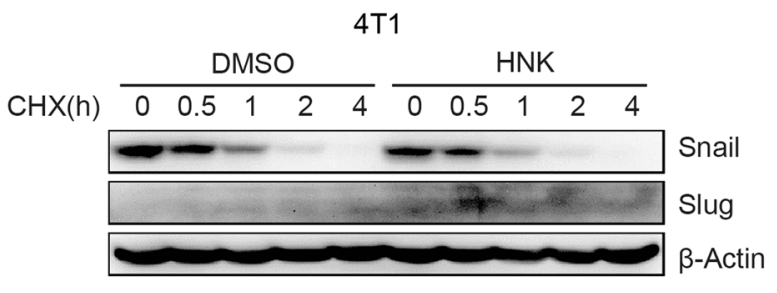

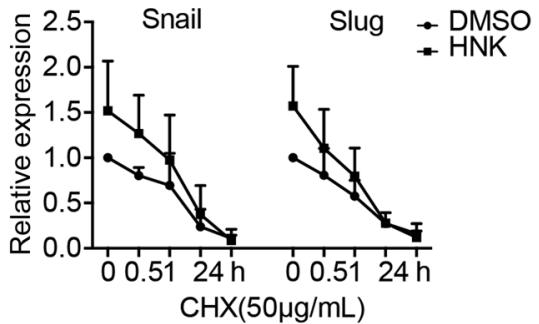
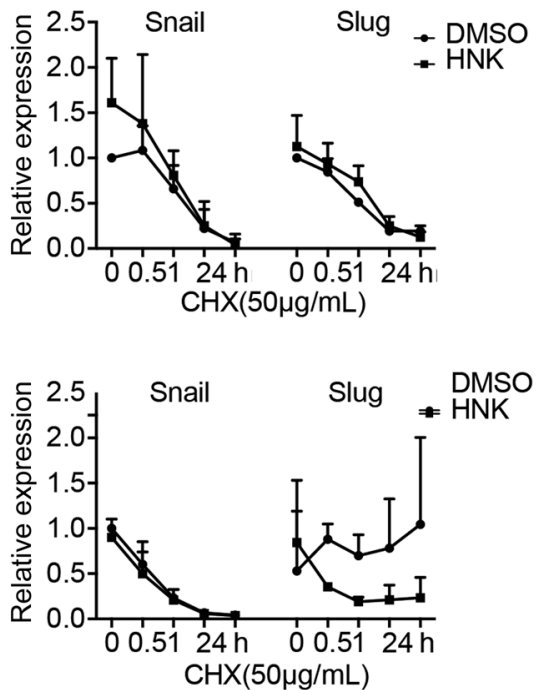

Fig. 5 Effect of HNK on Snail and Slug mRNA expression and protein stability. a Quantitative real-time PCR analysis of Snail and Slug mRNA expression following treatment with HNK for $0,6,12$ and $24 \mathrm{~h}$. The mRNA levels were normalized to GAPDH mRNA expression. The relative mRNA levels are shown as the fold change compared with untreated cells. The error bars represent the standard deviation. $\mathbf{b}$ The endogenous expression levels of Snail and Slug in MDA-MB-231, MCF7, and 4T1 cell lines. The relative expression levels were normalized to Snail of MDAMB-231 cells. c-e The protein stability of Snail and Slug expression was determined by collecting protein samples from cells treated with cycloheximide (CHX) $(50 \mu \mathrm{g} / \mathrm{mL})$ for $0,0.5,1,2$, and $4 \mathrm{~h}$, after pretreatment with HNK for $8 \mathrm{~h}$. ImageJ software was used to quantify the band densities of proteins. Quantification of western blot analysis and half-life relative to $\beta$-actin

ligase FBXL14 and Snail to increase Snail ubiquitination [40]. In contrast to these regulatory mechanisms, our data showed no change in the Snail or Slug mRNA levels following treatment with HNK. This result was confirmed with the data in GEO (GSE85871) deposited by Ponnurangam et al., which provide the gene expression profiles of MCF7 cells treated with HNK [41]. The stabilization of Snail and Slug protein expresssion measured by $\mathrm{CHX}$ chase experiments also supported the mRNA assay results in the presence of HNK.

Previous studies have suggested that many different stress signals cause the phosphorylation of elF $2 a$, which inhibits its translational function by reducing its affinity for ribosomes, thus attenuating mRNA translation [42, 43]. Importantly, HNK can induce the phosphorylation of elF2a in gastric cancer cells [34]. Our further research showed that HNK treatment resulted in increased elF2a phosphorylation prior to the decrease in Snail. The puromycin incorporation assay further demonstrated that HNK markedly inhibited Snail protein synthesis in MCF7 cells. These data suggest that HNK modulates Snail and Slug expression at the mRNA translation level and explained the interesting changes in Snail and Slug mRNA and protein expression.

In summary, our results demonstrate that HNK significantly inhibits EMT in breast cancer cells and reveal a novel mechanism by which HNK regulates Snail and Slug via downregulation of their protein translation (Fig. $6 \mathrm{c}$ ). These observations suggest that the HNK might be able to be developed as a suitable therapeutic strategy for breast cancer metastasis.

\section{ACKNOWLEDGEMENTS}

This research was supported by grants from the National Natural Science Foundation of China (81373437, 81702934, and 81621064), CAMS Innovation 
a

MCF7

MDA-MB-231

4T1
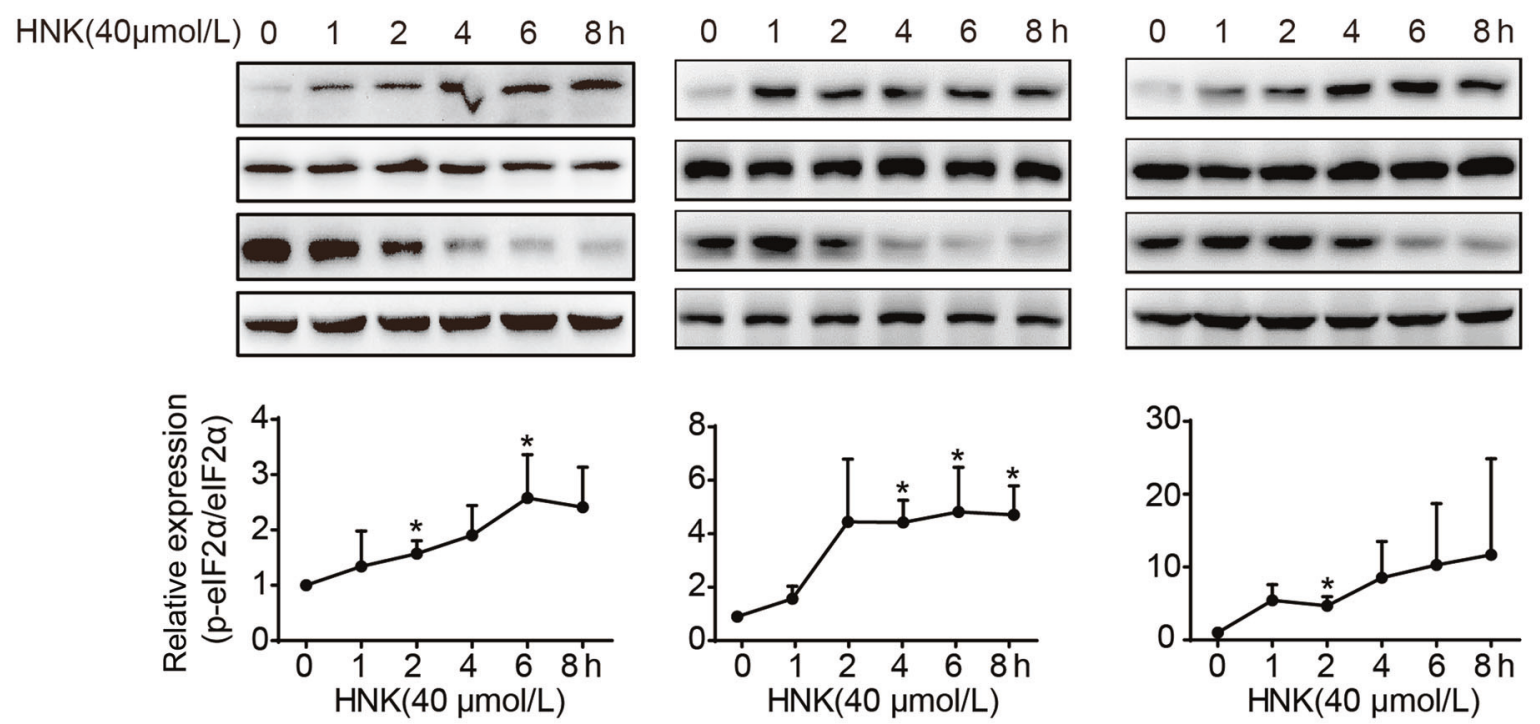

p-elF2 $\alpha$

elF $2 \alpha$

Snail
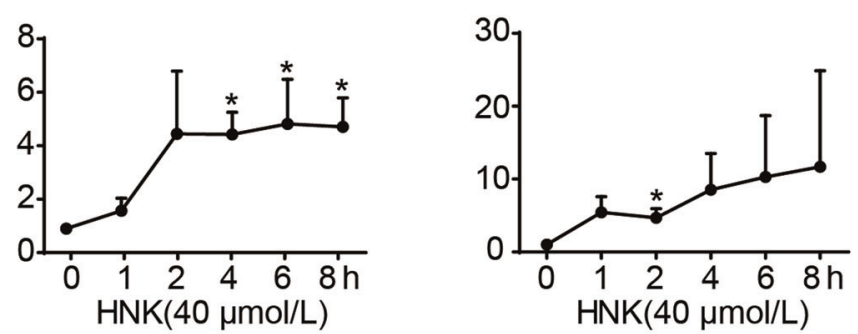

b

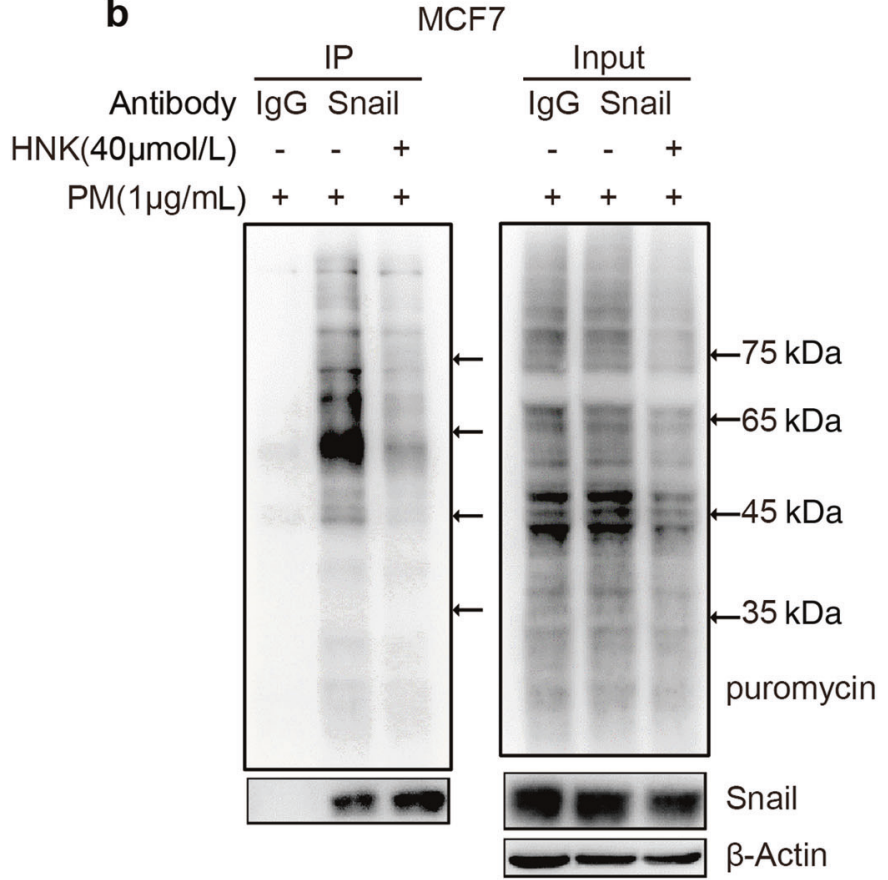

C

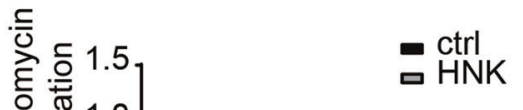

Fig. 6 HNK inhibits Snail protein translation in breast cancer cells. a Breast cancer cells were treated with HNK for different periods of time $(0$, $1,2,4,6$, and $8 \mathrm{~h}$ ) and were subjected to Western blotting assays. ImageJ software was used to calculate the band densities of proteins. ${ }^{*} P<$ 0.05 (IP) compared with the control group. b, $\mathbf{c}$ Breast cancer cells were cultured with HNK or DMSO for $8 \mathrm{~h}$ and puromycin (PM, $1 \mu \mathrm{mol} / \mathrm{L})$ was then added to the medium of cells for $1 \mathrm{~h}$ before harvesting. Cell extracts were immunoprecipitated (IP) with anti-Snail antibody the precipitated proteins and whole-cell extracts were immunoblotted using the indicated antibodies to measure puromycin incorporation. ImageJ software was applied to quantify the band densities of proteins. ${ }^{*} P<0.05$ compared with the control group. $\mathbf{d}$ Schematic of the proposed mechanism by which HNK inhibits EMT in breast cancer cells

Fund for Medical Sciences (CIFMS, 2016-12M-02-002), and "Significant New Drug Development" Major Science and Technology Development Projects of China (No. 2018ZX09711001-007-002).

\section{AUTHOR CONTRIBUTIONS}

SZC designed the study. WDW, YS and YL performed the animal and related studies. WDW conducted the other experiments and wrote the paper. SZC edited the paper. All the authors read and approved the final manuscript.

\section{ADDITIONAL INFORMATION}

Competing interests: The anthors declared that they have no conflicts of interest to this work.

\section{REFERENCES}

1. Siegel RL, Miller KD, Jemal A. Cancer statistics, 2018. CA Cancer J Clin. 2018;68:7-30.

2. DeSantis CE, Ma J, Goding Sauer A, Newman LA, Jemal A. Breast cancer statistics, 2017, racial disparity in mortality by state. CA Cancer J Clin. 2017;67:439-48. 
3. Mahalingaiah PK, Ponnusamy L, Singh KP. Chronic oxidative stress leads to malignant transformation along with acquisition of stem cell characteristics, and epithelial to mesenchymal transition in human renal epithelial cells. J Cell Physiol. 2015;230:1916-28.

4. Polyak K, Weinberg RA. Transitions between epithelial and mesenchymal states: acquisition of malignant and stem cell traits. Nat Rev Cancer. 2009;9:265-73.

5. Brabletz T, Kalluri R, Nieto MA, Weinberg RA. EMT in cancer. Nat Rev Cancer. 2018;18:128-34.

6. De Craene B, Berx G. Regulatory networks defining EMT during cancer initiation and progression. Nat Rev Cancer. 2013;13:97-110.

7. Gupta PB, Onder TT, Jiang G, Tao K, Kuperwasser C, Weinberg RA, et al. Identification of selective inhibitors of cancer stem cells by high-throughput screening. Cell. 2009;138:645-59.

8. Davis FM, Stewart TA, Thompson EW, Monteith GR. Targeting EMT in cancer: opportunities for pharmacological intervention. Trends Pharmacol Sci. 2014;35:479-88

9. Shibue T, Weinberg RA. EMT, CSCs, and drug resistance: the mechanistic link and clinical implications. Nat Rev Clin Oncol. 2017;14:611-29.

10. Zhang Y, Chen T, Yuan P, Tian R, Hu W, Tang Y, et al. Encapsulation of honokiol into self-assembled pectin nanoparticles for drug delivery to HepG2 cells. Carbohydr Polym. 2015;133:31-8.

11. Averett C, Bhardwaj A, Arora S, Srivastava SK, Khan MA, Ahmad A, et al. Honokiol suppresses pancreatic tumor growth, metastasis and desmoplasia by interfering with tumor-stromal cross-talk. Carcinogenesis. 2016;37:1052-61.

12. Zhang Q, Zhao W, Ye C, Zhuang J, Chang C, Li Y, et al. Honokiol inhibits bladder tumor growth by suppressing EZH2/miR-143 axis. Oncotarget. 2015;6:37335-48

13. Zhang $Y$, Ren $X$, Shi $M$, Jiang $Z$, Wang $H$, Su $Q$, et al. Downregulation of STAT3 and activation of MAPK are involved in the induction of apoptosis by HNK in glioblastoma cell line U87. Oncol Rep. 2014;32:2038-46.

14. Avtanski DB, Nagalingam A, Kuppusamy P, Bonner MY, Arbiser JL, Saxena NK, et al. Honokiol abrogates leptin-induced tumor progression by inhibiting Wnt1MTA1-beta-catenin signaling axis in a microRNA-34a dependent manner. Oncotarget. 2015;6:16396-410.

15. Liu SH, Lee WJ, Lai DW, Wu SM, Liu CY, Tien HR, et al. Honokiol confers immunogenicity by dictating calreticulin exposure, activating ER stress and inhibiting epithelial-to-mesenchymal transition. Mol Oncol. 2015;9:834-49.

16. Avtanski DB, Nagalingam A, Bonner MY, Arbiser JL, Saxena NK, Sharma D. Honokiol inhibits epithelial-mesenchymal transition in breast cancer cells by targeting signal transducer and activator of transcription 3/Zeb1/E-cadherin axis. Mol Oncol. 2014;8:565-80.

17. Bando T, Ishimaru Y, Kida T, Hamada Y, Matsuoka Y, Nakamura T, et al. Analysis of RNA-Seq data reveals involvement of JAK/STAT signalling during leg regeneration in the cricket Gryllus bimaculatus. Development. 2013;140:959-64.

18. Lv XQ, Qiao XR, Su L, Chen SZ. Honokiol inhibits EMT-mediated motility and migration of human non-small cell lung cancer cells in vitro by targeting c-FLIP. Acta Pharmacol Sin. 2016:37:1574-86.

19. Wang N, Wang Z, Nie S, Song L, He T, Yang S, et al. Biodegradable polymeric micelles coencapsulating paclitaxel and honokiol: a strategy for breast cancer therapy in vitro and in vivo. Int J Nanomed. 2017;12:1499-514.

20. Avtanski DB, Nagalingam A, Bonner MY, Arbiser JL, Saxena NK, Sharma D. Honokiol activates LKB1-miR-34a axis and antagonizes the oncogenic actions of leptin in breast cancer. Oncotarget. 2015;6:29947-62.

21. Wang $\mathrm{H}$, Wang $\mathrm{HS}$, Zhou $\mathrm{BH}$, Li CL, Zhang $\mathrm{F}$, Wang $X F$, et al. Epithelialmesenchymal transition (EMT) induced by TNF-alpha requires AKT/GSK-3betamediated stabilization of snail in colorectal cancer. PLoS ONE. 2013;8:e56664.

22. Sun M, Guo X, Qian X, Wang H, Yang C, Brinkman KL, et al. Activation of the ATM-Snail pathway promotes breast cancer metastasis. J Mol Cell Biol. 2012;4:304-15.

23. Cicchini C, Amicone L, Alonzi T, Marchetti A, Mancone C, Tripodi M. Molecular mechanisms controlling the phenotype and the EMT/MET dynamics of hepatocyte. Liver Int. 2015;35:302-10.
24. Li X, Chen H, Liu Z, Ye Z, Gou S, Wang C. Overexpression of MIST1 reverses the epithelial-mesenchymal transition and reduces the tumorigenicity of pancreatic cancer cells via the Snail/E-cadherin pathway. Cancer Lett. 2018:431:96-104.

25. Hao L, Ha JR, Kuzel P, Garcia E, Persad S. Cadherin switch from E- to N-cadherin in melanoma progression is regulated by the PI3K/PTEN pathway through Twist and Snail. Br J Dermatol. 2012;166:1184-97.

26. Xu M, Li J, Wang X, Meng S, Shen J, Wang S, et al. MiR-22 suppresses epithelialmesenchymal transition in bladder cancer by inhibiting Snail and MAPK1/Slug/ vimentin feedback loop. Cell Death Dis. 2018;9:209.

27. Fried LE, Arbiser JL. Honokiol, a multifunctional antiangiogenic and antitumor agent. Antioxid Redox Signal. 2009;11:1139-48.

28. Arora S, Singh S, Piazza GA, Contreras CM, Panyam J, Singh AP. Honokiol: a novel natural agent for cancer prevention and therapy. Curr Mol Med. 2012;12:1244-52.

29. Banerjee $P$, Basu A, Arbiser JL, Pal S. The natural product honokiol inhibits calcineurin inhibitor-induced and Ras-mediated tumor promoting pathways. Cancer Lett. 2013;338:292-9.

30. Yao CJ, Lai GM, Yeh CT, Lai MT, Shih PH, Chao WJ, et al. Honokiol eliminates human oral cancer stem-like cells accompanied with suppression of Wnt/ betacatenin signaling and apoptosis induction. Evid Based Complement Altern Med. 2013;2013:146136.

31. Kaufhold S, Bonavida B. Central role of Snail1 in the regulation of EMT and resistance in cancer: a target for therapeutic intervention. J Exp Clin Cancer Res. 2014;33:62.

32. Batlle E, Sancho E, Franci C, Dominguez D, Monfar M, Baulida J, et al. The transcription factor snail is a repressor of E-cadherin gene expression in epithelial tumour cells. Nat Cell Biol. 2000;2:84-9.

33. Cano A, Perez-Moreno MA, Rodrigo I, Locascio A, Blanco MJ, del Barrio MG, et al. The transcription factor snail controls epithelial-mesenchymal transitions by repressing E-cadherin expression. Nat Cell Biol. 2000;2:76-83.

34. Pan HC, Lai DW, Lan KH, Shen CC, Wu SM, Chiu CS, et al. Honokiol thwarts gastric tumor growth and peritoneal dissemination by inhibiting Tpl2 in an orthotopic model. Carcinogenesis. 2013;34:2568-79.

35. Cai J, Guan H, Fang L, Yang Y, Zhu X, Yuan J, et al. MicroRNA-374a activates Wnt/ beta-catenin signaling to promote breast cancer metastasis. J Clin Invest. 2013;123:566-79.

36. Chengye W, Yu T, Ping S, Deguang S, Keyun W, Yan W, et al. Metformin reverses bFGF-induced epithelial-mesenchymal transition in HCC cells. Oncotarget. 2017;8:104247-57.

37. Yuan JH, Yang F, Wang F, Ma JZ, Guo YJ, Tao QF, et al. A long noncoding RNA activated by TGF-beta promotes the invasion-metastasis cascade in hepatocellular carcinoma. Cancer Cell. 2014;25:666-81.

38. Iwasaki K, Ninomiya R, Shin T, Nomura T, Kajiwara T, Hijiya N, et al. Chronic hypoxia-induced slug promotes invasive behavior of prostate cancer cells by activating expression of ephrin-B1. Cancer Sci. 2018;109:3159-70.

39. Nguyen LT, Song YW, Cho SK. Baicalein inhibits epithelial to mesenchyma transition via downregulation of Cyr61 and LOXL-2 in MDA-MB231 breast cancer cells. Mol Cells. 2016;39:909-14.

40. Song L, Guo J, Chang R, Peng X, Li J, Xu X, et al. LKB1 obliterates Snail stability and inhibits pancreatic cancer metastasis in response to metformin treatment. Cancer Sci. 2018;109:1382-92.

41. Lv C, Wu X, Wang X, Su J, Zeng H, Zhao J, et al. The gene expression profiles in response to 102 traditional Chinese medicine (TCM) components: a general template for research on TCMs. Sci Rep. 2017;7:352.

42. Clavarino G, Claudio N, Couderc T, Dalet A, Judith D, Camosseto V, et al. Induction of GADD34 is necessary for dsRNA-dependent interferon-beta production and participates in the control of Chikungunya virus infection. PLoS Pathog. 2012;8: e1002708.

43. Han K, Jaimovich A, Dey G, Ruggero D, Meyuhas O, Sonenberg N, et al. Parallel measurement of dynamic changes in translation rates in single cells. Nat Methods. 2014;11:86-93. 